Reprod. Nutr. Dévelop., 1985, 25 (1 A), 17-32.

\title{
Effet du génotype, de l'âge et de la saison sur les follicules préovulatoires de la lapine 8 heures après la saillie
}

\author{
Françoise HULOT, J.-C. MARIANA (*) \\ avec la collaboration technique de G. GATTIAU
}

Station d'Amélioration génétique des Animaux, I.N.R.A. B.P. 27, 31326 Castanet Tolosan, France.

(*) Station de Physiologie de la Reproduction, I.N.R.A. Nouzilly, 37380 Monnaie.

Summary. Effect of genotype, age and season on preovulatory follicles of the doe-rabbit 8 hours after mating.

The purpose of this study was to compare the population of preovulatory follicles in two INRA strains of rabbits of different natural ovulation rates. The two strains chosen were the Californian (A1066) with an ovulation rate of 13 and the New Zealand (A1077) with an ovulation rate of 11 .

Nulliparous, primiparous and multiparous does were followed during the four seasons. Eight hours after mating the right ovaries of 154 of these animals were collected.

The following data were recorded : the number of follicles with an area equal to or more than $5.10^{5} \mu \mathrm{m}^{2}$ (diameter : $0.8 \mathrm{~mm}$ ), the area of that section of the follicle containing the oocyte, and the presence of pyknotic cells with granulosa.

The higher ovulation rate of Californian does was due to a significantly higher number of healthy follicles. The mean size of these follicles, their area (between $5.10^{5} \mu \mathrm{m}^{2}$ and $3.10^{6}$ $\mu \mathrm{m}^{2}$ ) distribution and rate of atresia were similar in the two strains.

Environmental factors led to changes in the number and size of healthy follicles which were responsible for the number of ovulations, while the rate of atresia remained constant. The seasonal effect was most marked in November by a definite reduction in follicle size, particularly in multiparous does.

Follicle number and size increased with parity number ; this seemed to be due to an acceleration of the growth rate and to the increasing number of large-sized follicles.

As there were no interactions between genotype and environmental factors, it can be concluded that the latter modified follicular growth parameters in the same way in both strains and that these strains can be identified in all cases on the basis of their ovarian characteristics.

About $1 / 3$ of the does exhibited almost exclusively healthy follicles, while in the remaining $2 / 3$ both healthy and atretic follicles were seen. These proportions were the same in both female genotypes. Those possessing only healthy follicles also had the largest ones. It is suggested that atresia could be a regulatory factor of ovulation rate along with the preovulatory level of FSH.

$23 \%$ of the does accepting the buck showed no oocytes in meiosis, even though their follicle size exceeded $5.10^{5} \mu \mathrm{m}^{2}$. The mean number of these follicles was the same as that of does with oocytes in meiosis, but they were half as large. It is suggested that the follicle oestrogen secretion of these does was not high enough to induce ovulatory release of LH at mating. 


\section{Introduction.}

Bien que de prolificité comparable, les lapines de souches INRA A 1066 d'origine Californienne et A 1077 d'origine Néo-Zélandaise diffèrent par le niveau de l'ovulation et par le taux de mortalité embryonnaire ; la lapine Californienne présente en moyenne deux ovulations de plus, mais une moins bonne survie embryonnaire. Ces caractères sont susceptibles d'évoluer selon la parité et la saison (Hulot et Matheron, 1981).

Les différences de taux d'ovulation entre souches peuvent résulter de l'importance relative du soutien gonadotrope, niveau de base et décharges ovulantes, permettant ou non l'ovulation de tous les follicules mûrs ou d'un nombre fondamentalement différent de follicules préovulatoires au moment de l'accouplement. C'est cette seconde hypothèse que nous avons choisie de tester en étudiant la taille et l'état des follicules préovulatoires ainsi que le taux d'ovulation enregistré au $12^{\mathrm{e}}$ jour de gestation, ce qui permet de connaître en même temps, la mortalité embryonnaire.

\section{Protocole expérimental.}

Les femelles faisant l'objet de cette étude sont contemporaines, et pour la majorité d'entre elles, pleines sœurs des lapines utilisées dans l'expérimentation Hulot et Matheron, 1981.

Chacune des souches a été étudiée dans trois états physiologiques : nullipare (20 semaines), primipare et multipare (après la $3^{\mathrm{e}}$ portée). Cette étude a eu lieu à quatre moments de l'année, choisis pour étudier l'effet de la saison : février (saison 1), mai (saison 2), août (saisson 3) et novembre (saison 4). 154 femelles au total ont été utilisées. Elles ont été saillies et sacrifiées $8 \mathrm{~h}$ post-coïtum, stade où la reprise de la méiose est bien visible dans les ovocytes des follicules sensibles à la décharge de LH (Cherney et al., 1975 ; Thibault et al., 1976). L'ovaire droit est prélevé, fixé dans du Bouin Hollande pendant 3 jours. Après déshydratation et inclusion dans la cytoparaffine, des coupes sériées de $10 \mu \mathrm{m}$ sont réalisées et colorées à I'hématoxyline de Regaud. La population étudiée est celle des plus gros follicules supposés être les follicules préovulatoires.

Le choix de la mesure est la surface car c'est un critère global tenant compte à la fois du nombre de cellules et de la taille de l'antrum (Mauléon et Rao, 1963). La surface du follicule, délimité par sa lame basale, est planimétrée à l'aide d'une table digitale Leitz, au niveau de la coupe contenant le nucléole de l'ovocyte ou les chromosomes si la méiose a repris. Cette méthode peut être appliquée au follicule de la lapine dont l'ovocyte occupe une position subcentrale. Sur cette même section est estimée la qualité du follicule par détection des cellules pycnotiques de la granulosa ou détachées dans la lumière de l'antrum. La limite de la définition de l'atrésie a été fixée à 5 cellules pycnotiques par section (Mariana, 1984).

Les nombres moyens de follicules ont été comparés par une analyse de variance à 3 facteurs avec interaction : génotype, parité, saison (Harvey, 1975). La comparaison des taux d'atrésie a été réalisée par la méthode du $X^{2}$. 
Les follicules ont été regroupés en 10 classes de taille fixées arbitrairement dont les limites sont en progression géométrique de raison 1,23. Les correspondances entre les numéros de classe, la surface et le diamètre équivalent (DE) sont les suivantes (tabl. 1).

TABLEAU 1

Correspondance entre les numéros de classe, la surface et le diamètre équivalent (DE) des follicules.

\begin{tabular}{ccc}
\hline Numéro de classe & Surface & Diamètre équivalent \\
\hline 1 & $500000-615572 \mu \mathrm{m}^{2}$ & $0.80-0.85 \mathrm{~mm}$ \\
2 & $615573-757858 \mu \mathrm{m}^{2}$ & $0.85-1.00 \mathrm{~mm}$ \\
3 & $757859-933033 \mu \mathrm{m}^{2}$ & $1.00-1.10 \mathrm{~mm}$ \\
4 & $933034-1148698 \mu \mathrm{m}^{2}$ & $1.10-1.20 \mathrm{~mm}$ \\
5 & $1148699-1414213 \mu \mathrm{m}^{2}$ & $1.20-1.35 \mathrm{~mm}$ \\
6 & $1414213-1741101 \mu \mathrm{m}^{2}$ & $1.35-1.50 \mathrm{~mm}$ \\
7 & $1741101-2143546 \mu \mathrm{m}^{2}$ & $1.50-1.65 \mathrm{~mm}$ \\
8 & $2143546-2639016 \mu \mathrm{m}^{2}$ & $1.65-1.85 \mathrm{~mm}$ \\
9 & $2639016-3249000 \mu \mathrm{m}^{2}$ & $1.85-2.00 \mathrm{~mm}$ \\
10 & $3249000-4000000 \mu \mathrm{m}^{2}$ & $2.00-2.25 \mathrm{~mm}$ \\
\hline
\end{tabular}

Les distributions de taille des follicules ainsi obtenues ont été comparées soit par le test de Kolmogorov Smirnov (KS) soit par des analyses de table de contingence au moyen du test 21 (Sokal et Rohlf, 1969). Une analyse des correspondances entre les différents génotypes, les périodes d'études, les parités et les classes de taille a permis d'avoir une vision globale et préliminaire des associations les plus fortes.

En outre, 56 lapines de chaque souche représentant un échantillon moyen de la population des femelles étudiées aux trois stades précédemment définis ont été abattues 12 jours après la mise au mâle pour connaître le taux d'ovulation et le nombre de fœtus.

\section{Résultats.}

1) Taux d'ovulation.

La distribution des taux d'ovulation est unimodale dans les 2 souches (fig. 1) et le nombre d'ovulation moyen est de 11,00 $\pm 0,38$ chez la Néozélandaise et
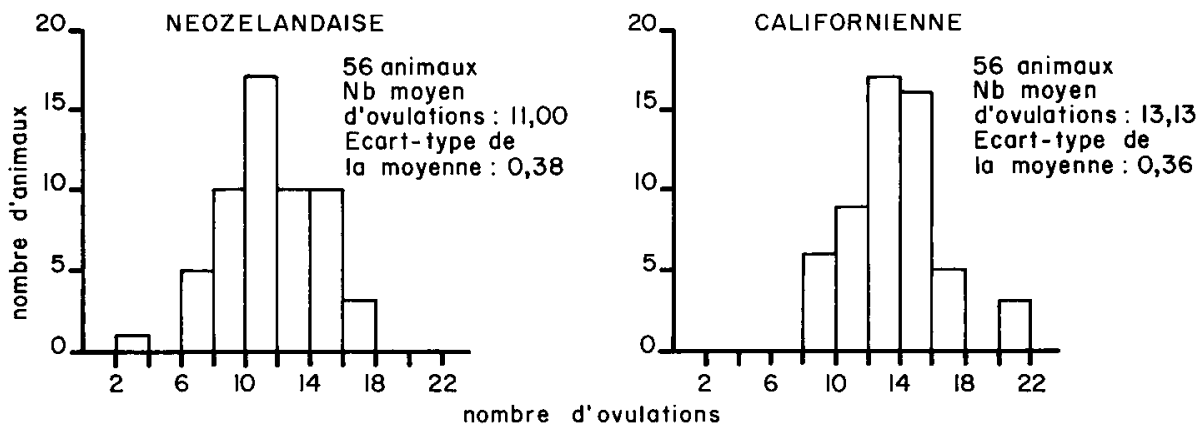

FIG. 1. - Distribution des nombres d'ovulations dans les deux souches de femelles ovulant. 
$13,13 \pm 0,36$ chez la Californienne ; on retrouve donc bien la différence de 2 ovulations au profit des femelles de souche Californienne.

Bien que ceci ne soit pas une preuve, le caractère unimodal des distributions est un argument en faveur du fait que chacune des souches est homogène, et qu'il n'existe pas de sous-population de femelles à taux d'ovulation, soit particulièrement élevé, soit particulièrement faible ; l'écart-type des valeurs est de plus le même.

\section{2) Follicules préovulatoires.}

Huit heures après le coït, la surface minimale d'un follicule dont l'ovocyte a repris la méiose est d'environ $500000 \mu \mathrm{m}^{2}$ (DE : 0,8 mm). Le noyau de l'ovocyte est alors au stade anaphase-télophase de la première division de maturation ou a expulsé le premier globule polaire.

II existe deux lots de femelles:

- un lot A de 118 femelles : tous les follicules ovariens égaux ou supérieurs à une surface de $500000 \mu \mathrm{m}^{2}$ ont des ovocytes dont le noyau reprend la méiose. Certains follicules dont la granulosa présente des signes de pycnose incontestables possèdent des ovocytes reprenant leur méiose dans des conditions apparemment normales.

- un lot B de 36 femelles : aucun follicule ayant la surface minimale précisée cidessus ne possède $d^{\prime}$ ovocytes reprenant la méiose (bien que ces femelles aient accepté l'accouplement).

\section{3) Nombres moyens de follicules sains et atrétiques.}

Lot $A$ : Le nombre moyen de follicules de diamètre supérieur à $0,8 \mathrm{~mm}$ est de 6,77 par ovaire (tabl. 2) répartis en follicules sains $(5,49)$ et en follicules atrétiques $(1,28)$. Le taux d'atrésie global (nombre de follicules atrétiques/nombre de follicules totaux) est de $19 \%$.

Lot $B$ : Le nombre moyen de l'ensemble des follicules sains + atrétiques de diamètre supérieur à $0,8 \mathrm{~mm}$ est de 6,69 comparable à celui du Lot $A$. Le nombre moyen de follicules sains de 4,67 et le taux d'atrésie de $30 \%$ diffèrent significativement des valeurs correspondantes du Lot $A$.

3.1. Comparaison entre les deux génotypes (parité et saison confondues). - Dans le Lot $A$, les moyennes du nombre de follicules diffèrent significativement entre les deux souches; les femelles Californiennes ont en moyenne 7,81 follicules totaux et 6,50 follicules sains par ovaire ; les Néo-Zélandaises en ont respectivement 5,95 et 4,70 (tabl. 2). Les nombres de follicules atrétiques un peu plus élevés chez la Néo-Zélandaise ne sont pas significativement différents de ceux de la Californienne.

Dans le Lot $B$, les nombres moyens de follicules totaux, sains et atrétiques sont plus élevés chez la Californienne. Toutefois, les différences ne sont statistiquement pas significatives.

Dans les analyses ultérieures, les femelles du lot B ne seront pas prises en compte, leur effectif étant trop faible. 
TABLEAU 2

Nombres moyens et écart-type (s) des follicules normaux, atrétiques, normaux + atrétiques, en fonction du génotype, de la parité et de la saison.

Follicules normaux

Follicules atrétiques

Ensemble des follicules (normaux + atrétiques)

$\mathrm{Nb}$. \& Nb. total $\begin{gathered}\mathrm{Nb} \text {. } \\ \text { moyen }\end{gathered} \mathrm{s} \quad \mathrm{Nb}$. total $\begin{gathered}\mathrm{Nb} . \\ \text { moyen }\end{gathered} \mathrm{s} \underset{\text { atrésie }}{\mathrm{Taux}} \mathrm{Nb}$. total $\begin{gathered}\mathrm{Nb} \text {. } \\ \text { moyen }\end{gathered}$

\begin{tabular}{|c|c|c|c|c|c|c|c|c|c|c|c|c|}
\hline A & Total & 118 & 648 & 5,49 & 2,13 & 151 & 1,28 & 1,38 & 0,19 & 799 & 6,77 & 2,52 \\
\hline \multirow[t]{2}{*}{ B } & Total & 36 & 168 & 4,67 & 2,39 & 73 & 2,03 & 1,52 & 0,30 & 241 & 6,69 & 2,77 \\
\hline & Test & & & * & & & * & & ** & & NS & \\
\hline Lot & Cal. & 52 & 338 & 6,50 & 1,72 & 68 & 1,31 & 1,45 & 0,17 & 406 & 7,81 & 2,14 \\
\hline \multirow[t]{2}{*}{$A$} & $\mathrm{NZ}$ & 66 & 310 & 4,70 & 2,10 & 83 & 1,26 & 1,35 & 0,21 & 393 & 5,95 & 2,50 \\
\hline & Test & & & ** & & & NS & & NS & & $* *$ & \\
\hline Lot & Cal. & 20 & 102 & 5,10 & 2,65 & 48 & 2,40 & 1,53 & 0,32 & 150 & 7,50 & 2,64 \\
\hline \multirow[t]{2}{*}{ B } & $\mathrm{NZ}$ & 16 & 66 & 4,12 & 1,96 & 25 & 1,56 & 1,41 & 0,27 & 91 & 5,69 & 2,65 \\
\hline & Test & & & NS & & & NS & & NS & & NS & \\
\hline Lot & Null. & 45 & 224 & 4,98 & 2,05 & 56 & 1,24 & 1,33 & 0,20 & 280 & 6,22 & 2,58 \\
\hline \multirow[t]{3}{*}{$A$} & Primip. & 40 & 225 & 5,63 & 2,19 & 52 & 1,30 & 1,45 & 0,19 & 277 & 6,93 & 2,47 \\
\hline & Multip. & 33 & 199 & 6,03 & 2,08 & 43 & 1,30 & 1,42 & 0,18 & 242 & 7,33 & 2,42 \\
\hline & Test & & & $\begin{array}{l}\text { NS } \\
* 10 \%\end{array}$ & & & NS & & NS & & NS & \\
\hline Lot & S1 & 27 & 153 & 5,67 & 2,27 & 38 & 1,41 & 1,67 & 0,20 & 191 & 7,07 & 2,53 \\
\hline \multirow[t]{4}{*}{$A$} & S2 & 34 & 187 & 5,50 & 1,93 & 46 & 1,35 & 1,30 & 0,20 & 233 & 6,85 & 2,55 \\
\hline & \$3 & 30 & 167 & 5,57 & 2,21 & 40 & 1,33 & 1,40 & 0,19 & 207 & 6,90 & 2,38 \\
\hline & S4 & 27 & 141 & 5,22 & 2,26 & 27 & 1,00 & 1,21 & 0,16 & 168 & 6,22 & 2,68 \\
\hline & Test & & & NS & & & NS & & NS & & NS & \\
\hline
\end{tabular}

Lot A : Fernelles dont les ovocytes reprennent la méiose ; Lot B : Femelles dont les ovocytes ne reprennent pas la méiose.

S1: février; $\mathrm{S2}$ : mai ; $\mathrm{S3}$ : août ; $\mathrm{S4}$ : novembre.

[Les interactions " diverses " sont non significatives (NS).]

3.2. Comparaison entre les parités (génotype et saison confondus). - Les nombres moyens de follicules totaux et normaux augmentent d'environ 1 follicule (valeur significative à $10 \%$ ) entre les stades nullipare et multipare (tabl. 2). Les nombres moyens de follicules atrétiques ne sont pas significativement différents pour les trois parités. II n'y a pas d'interaction génotype-parité : la femelle Califor- 
nienne à chaque stade de sa vie, possède plus de follicules normaux et un peu moins de follicules atrétiques que la Néo-Zélandaise.

3.3. Comparaison entre les saisons (parité et génotype confondus). - II existe des fluctuations saisonnières entre les nombres moyens de follicules totaux, normaux (baisse en novembre) et atrétiques mais elles ne sont pas significatives.

II $n^{\prime} y$ a pas d'interaction génotype-saison : chacun des génotypes conserve ses caractéristiques propres au cours de l'année.

\section{4) Tailles moyennes des follicules sains et atrétiques.}

La taille des follicules dont les ovocytes ont repris leur méiose est très variable : elle est comprise entre 500000 et $3000000 \mu \mathrm{m}^{2}$ environ (DE : 0,8 à $2 \mathrm{~mm}$ ) ; aussi, convient-il de les ordonner selon des classes de taille pour analyser leur distribution.

Dans le lot A, la population des follicules sains (648) est bien délimitée, réduite dans les classes extrêmes, dessinant un mode important légèrement déplacé vers les grandes tailles (classes 5, 6, 7) (fig. 2). Leur taille moyenne est de $1519000 \mu \mathrm{m}^{2}$ (DE : 1,4 mm) (tabl. 3). Les follicules atrétiques (151) sont significativement plus petits (surface moyenne de $1271000 \mu \mathrm{m}^{2} ; \mathrm{DE}=1,2 \mathrm{~mm}$ ). Le taux d'atrésie décroît quand la taille des follicules augmente.

Dans le groupe B, la distribution des follicules sains (168) est significativement différente : elle se situe essentiellement dans les 4 premières classes de petits follicules et s'amenuise jusqu'à la classe 7. La proportion de follicules atrétiques (73) est sensiblement égale dans ces 4 classes puis décroît. Les surfaces moyennes des follicules normaux et atrétiques sont peu différentes.
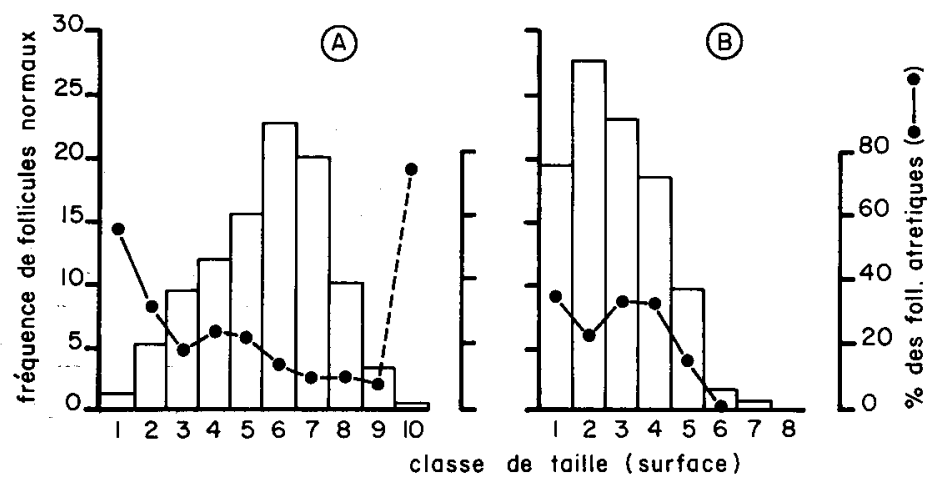

FIG. 2. - Distribution des follicules normaux en classes de taille $\left(\mu \mathrm{m}^{2}\right)$ pour /'ensemble des femelles. Evolution du pourcentage d'atrésie en fonction de la taille des follicules. Lot $A$ : femelles dont les ovocytes reprennent la méiose (toutes femelles confondues); Lot B : femelles dont les ovocytes ne reprennent pas la méiose.

4.1. Comparaison entre les deux génotypes (tabl. 3, fig. 3). - Dans le lot A, la distribution des follicules sains en classes de taille est tout à fait comparable entre les deux génotypes et présente de ce fait, des caractéristiques similaires à celles de la population générale. Les surfaces moyennes des follicules sont remar- 
TABLEAU 3

Surfaces moyennes et écart-type (s) des follicules normaux et atrétiques en fonction du génotype, de la parité et de la saison.

\begin{tabular}{|c|c|c|c|c|c|c|c|c|}
\hline & & \multicolumn{5}{|c|}{ Follicules normaux } & \multicolumn{2}{|l|}{ Follicules atrétiques } \\
\hline & & Nb. & $\mathrm{Nb}$. & Surf. moyenne (1) & s & $\mathrm{Nb}$ & Surf. moyenne (1) & $\mathbf{s}$ \\
\hline A & Total & 118 & 648 & 1519263 & 548355 & 151 & 1270931 & 596292 \\
\hline \multirow[t]{2}{*}{$B$} & Total & 36 & 168 & 836060 & 243479 & 73 & 795273 & 184377 \\
\hline & \multicolumn{2}{|c|}{ Test } & \multicolumn{3}{|c|}{$* *$} & \multicolumn{3}{|c|}{$* *$} \\
\hline \multirow{3}{*}{ Lot $A$} & Cal. & 52 & 338 & 1520426 & 519565 & 68 & 1232766 & 483924 \\
\hline & $\mathrm{NZ}$ & 66 & 310 & 1518023 & 579007 & 83 & 1302210 & 675626 \\
\hline & \multicolumn{2}{|c|}{ Test } & \multicolumn{3}{|c|}{ NS } & \multicolumn{3}{|c|}{ NS } \\
\hline \multirow{3}{*}{ Lot B } & Cal. & 20 & 102 & 830202 & 225206 & 48 & 824745 & 196674 \\
\hline & $\mathrm{NZ}$ & 16 & 66 & 845119 & 270866 & 25 & 738688 & 145476 \\
\hline & \multicolumn{2}{|c|}{ Test } & \multicolumn{3}{|c|}{ NS } & \multicolumn{3}{|c|}{ * } \\
\hline & Nullipare & 45 & 224 & 1476501 & 503056 & 56 & 1276625 & 486756 \\
\hline \multirow[t]{3}{*}{ Lot $A$} & Primipare & 40 & 225 & 1448630 & 525603 & 52 & 1223526 & 713771 \\
\hline & Multipare & 33 & 199 & 1647355 & 600714 & 43 & 1320863 & 579289 \\
\hline & \multicolumn{2}{|c|}{ Test } & \multicolumn{3}{|c|}{$* *$} & \multicolumn{3}{|c|}{ ** } \\
\hline \multirow{4}{*}{ Lot $A$} & Saison 1 & 27 & 153 & 1579504 & 624467 & 38 & 1276855 & 719627 \\
\hline & Saison 2 & 34 & 187 & 1521042 & 470106 & 46 & 1341259 & 578414 \\
\hline & Saison 3 & 30 & 167 & 1596462 & 624257 & 40 & 1286651 & 577838 \\
\hline & Saison 4 & 27 & 141 & 1360228 & 417296 & 27 & 1119527 & 448867 \\
\hline \multicolumn{3}{|c|}{ Test } & & ** & & \multicolumn{3}{|c|}{ NS } \\
\hline
\end{tabular}

Lot A : Femelles dont les ovocytes reprennent la méiose ; Lot B : Femelles dont les ovocytes ne reprennent pas la méiose.

(1) moyenne surface $=\mu \mathrm{m}^{2}$.

Cal. : Californienne ; NZ: Néo-Zélandaise.

Saison 1 : février ; Saison 2 : mai ; Saison 3 : août ; Saison 4 : novembre.

[L'interaction saison-parité sur les follicules normaux est hautement significative $\left(^{* *}\right.$ ) ; les autres interactions « diverses » sont non significatives (NS).]

quablement identiques dans les deux souches (1518 000 et $1520000 \mu \mathrm{m}^{2}$ ) (DE : $1,4 \mathrm{~mm}$ ). Les profils moyens des taux d'atrésie sont également comparables pour les deux génotypes. Le taux d'atrésie est significativement plus important pour les follicules de petite taille dans les deux souches et décroît avec la taille du follicule.

Dans le lot $B$, les distributions de follicules sains sont comparables dans les deux souches, caractérisées comme dans la population générale par un grand nombre de follicules dans les classes de petites tailles. Les surfaces moyennes 


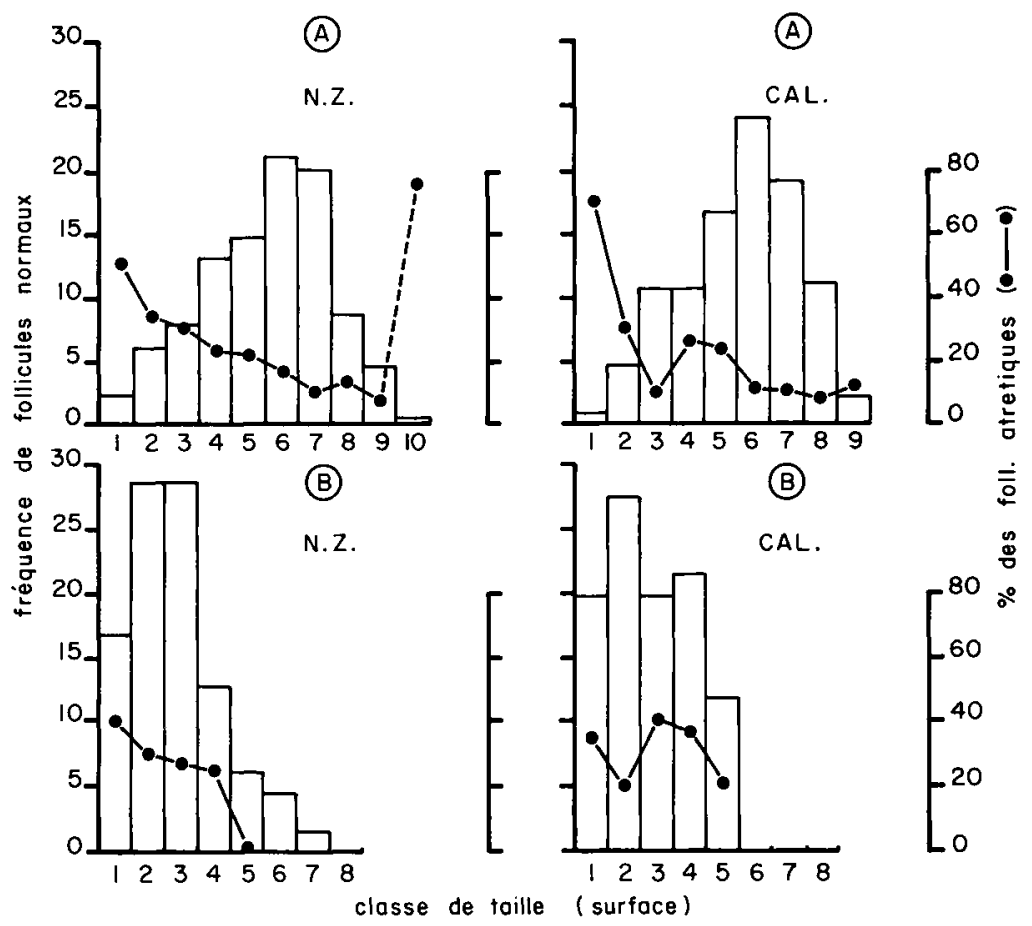

FIG. 3. - Distribution des follicules normaux chez la souche Néo-Zélandaise et Californienne (lot $A$ et lot B : voir légende fig. 2). Evolution du pourcentage d'atrésie en fonction de la taille des follicules.

sont comparables pour les follicules normaux (environ $830000 \mu \mathrm{m}^{2} ; \mathrm{DE}$ : $1,05 \mathrm{~mm}$ ), plus petites chez les Néo-Zélandaises pour les follicules atrétiques.

4.2. Influence de la parité (tabl. 3, fig. 4). - La taille moyenne des follicules sains et atrétiques diffère significativement pour les nullipares, primipares et multipares; ceci se traduit très clairement au niveau des distributions; celles des

NULLIPARES
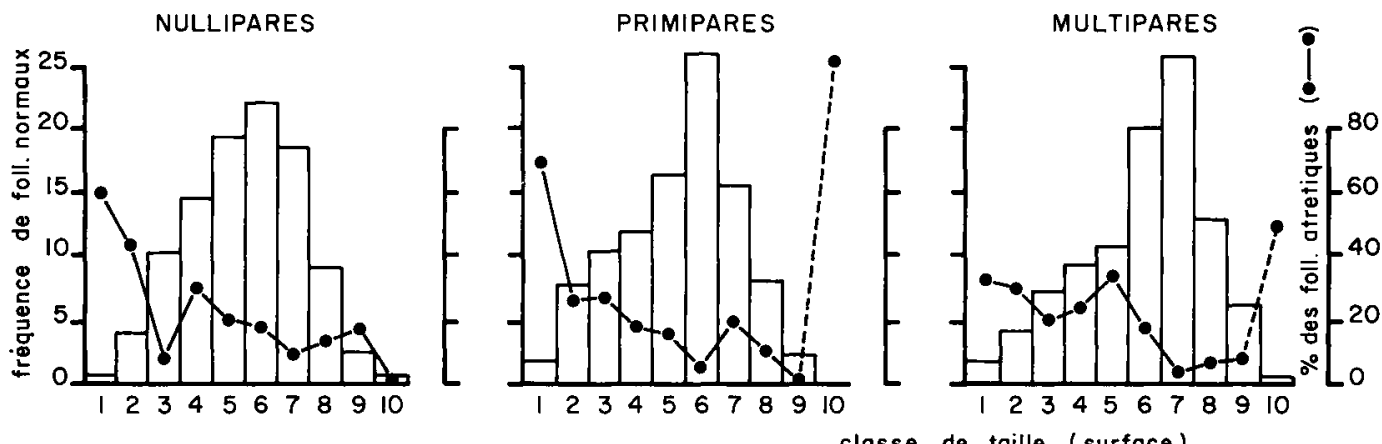

FIG. 4. - Distribution des follicules normaux en fonction de la parité (Néo-Zélandaise + Californienne). Evolution du pourcentage d'atrésie en fonction de la taille des follicules. 
femelles nullipares et primipares sont comparables avec un fort pourcentage de follicules normaux en classes 5, 6 et 7 ; celles des multipares présentent un mode plus dissymétrique avec un tassement des follicules vers les grandes tailles $6,7,8$ et 9 ; la présence de la classe 10 est négligeable ( 1 follicule).

Les profils des taux d'atrésie ne diffèrent pas selon la parité. Le taux d'atrésie décroît quand la taille des follicules augmente; les très gros follicules (classe 10) font exception.

4.3. Influence de la saison (tabl. 3 ; fig. 5). - La taille moyenne des follicules normaux diffère selon les saisons. Au mois de novembre (saisont 4) on observe une réduction significative de la taille des follicules par rapport aux autres saisons, due notamment à une forte réduction de la classe 8 et à la disparition des classes de grandes tailles 9 et 10 .

Les profils du taux d'atrésie ne sont pas significativement différents d'une saison à l'autre.

Les interactions diverses génotype-parité et génotype-saison ne sont pas significatives: Quelle que soit la parité ou la saison considérée, la distribution des follicules en classe de taille n'est pas significativement différente pour les Californiennes et les Néo-Zélandaises.

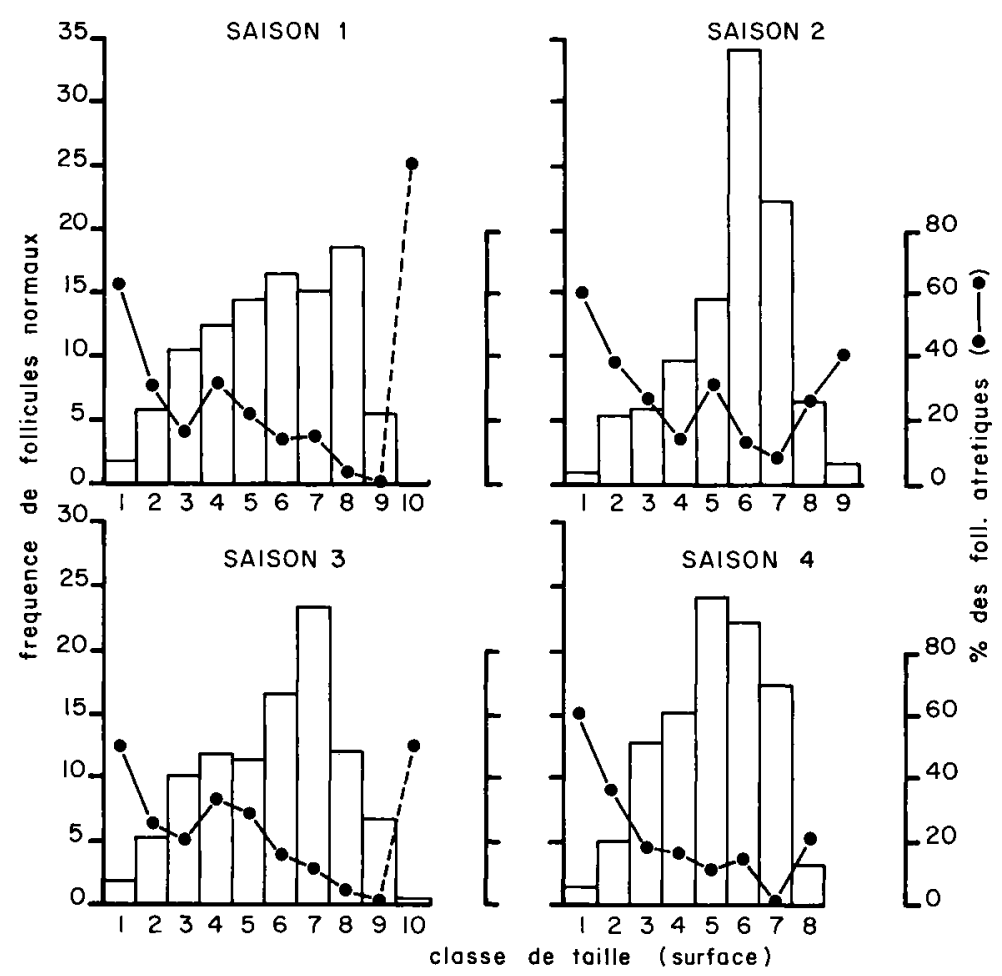

FIG. 5. - Distribution des follicules normaux en fonction des 4 saisons (Néo-Zélandaise + Californienne) S1: février ; S2 : mai ; S3 : août, S4 : novembre. Evolution du pourcentage d'atrésie en fonction de la taille des follicules. 
4.4. Interaction parité-saison. - L'analyse factorielle des correspondances (fig. 6) où les critères d'analyses sont les nombres de follicules répartis en 5 classes de tailles (les 10 classes sont regroupées 2 à 2 ) et les facteurs étudiés sont la parité et le génotype, montre qu'on peut distinguer, pour les deux souches, deux groupes de femelles: d'une part, les nullipares et les primipares des quatre saisons, les multipares de la saison 4, d'autre part les multipares des saisons 1, 2 et 3. Ces dernières, dans les deux souches, sont en effet caractérisées par la présence d'un nombre plus important de gros follicules dont les diamètres sont compris entre 1,5 et $2,25 \mathrm{~mm}$.

L'analyse de variance (tabl. 3) réalisée en prenant comme critère la taille des follicules indique que l'interaction parité-saison est significative; ceci confirme l'association mise en évidence par l'analyse factorielle des correspondances.

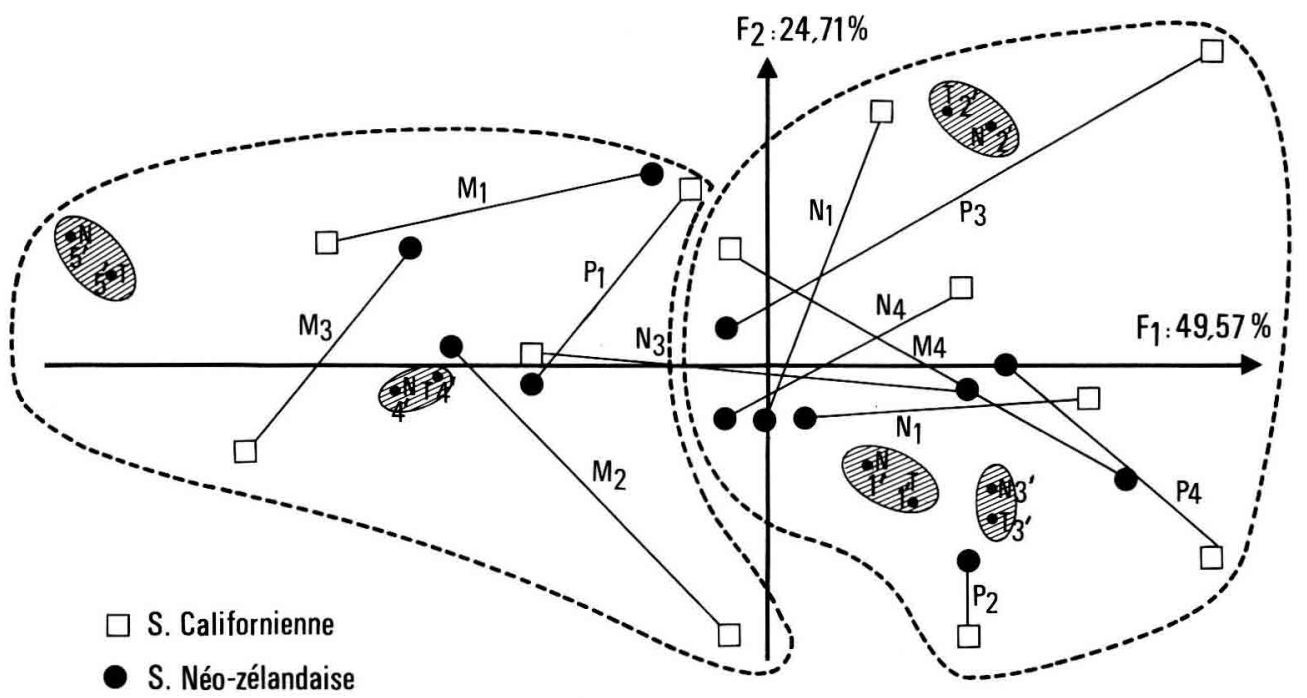

FIG. 6. - Analyse factorielle des correspondances du nombre total de follicules (jT) ou du nombre des follicules normaux (jN) appartenant à la classe de taille $j$ (nouvelles classes regroupées) où $\mathrm{j}=1^{\prime}, 2^{\prime}, 3^{\prime}, 4^{\prime}, 5^{\prime}$.

classe $1^{\prime}: \quad 500000-757858 \mu \mathrm{m}^{2} \quad 0.80-1.00 \mathrm{~mm}$ Diamètre équivalent

$2^{\prime}: 757859-1148698 \mu \mathrm{m}^{2} \quad 1.00-1.20 \mathrm{~mm}$ Diamètre équivalent

$3^{\prime}: 1148699-1741101 \mu \mathrm{m}^{2} \quad 1.20-1.50 \mathrm{~mm}$ Diamètre équivalent

$4^{\prime}: 1741102-2639016 \mu \mathrm{m}^{2} \quad 1.50-1.85 \mathrm{~mm}$ Diamètre équivalent

$5^{\prime}: 2639016-4000000 \mu \mathrm{m}^{2} \quad 1.85-2.25 \mathrm{~mm}$ Diamètre équivalent

$\mathrm{N}_{\mathrm{i}}$ : Nullipares de la saison $\mathrm{i}$.

$P_{i}$ : Primipares de la saison $\left.i\right\} \quad$ où $i=1,2,3,4$

$\mathrm{M}_{\mathrm{i}}$ : Multipares de la saison $\mathrm{i}$

Les femelles californiennes, Néo-Zélandaises de la même saison sont représentées par le symbole i ; $N_{i}$ représente par exemple les nullipares de la saison i.

5) Ovaires présentant ou non de l'atrésie (tabl. 4). - Parmi les 118 femelles du lot A, $37 \%$ ne présentent aucun signe d'atrésie parmi les follicules de plus de $500000 \mu \mathrm{m}^{2}$ et $63 \%$ ont un mélange de follicules atrétiques et de follicules nor- 


\section{TABLEAU 4}

Nombres moyens et surfaces moyennes des follicules normaux et atrétiques dans les deux catégories $d^{\prime}$ ovaires (ovaires à follicules sains uniquement, ovaires à follicules sains et atrétiques), en fonction du génotype, de la parité et de la saison.

\begin{tabular}{|c|c|c|c|c|c|c|c|c|c|c|c|}
\hline & \multicolumn{4}{|c|}{ Ovaire à follicules sains } & \multicolumn{7}{|c|}{ Ovaire à follicules sains et atrétiques } \\
\hline & $\begin{array}{l}\text { p. } 100 \\
\text { (1) }\end{array}$ & $\begin{array}{c}\mathrm{Nb} . \\
\text { foll. } \\
\text { norm. }\end{array}$ & $\begin{array}{l}\text { Moy. } \\
\text { nb. } \\
\text { foll. }\end{array}$ & $\begin{array}{c}\text { Moy. } \\
\text { surface } \\
\text { (2) }\end{array}$ & $\begin{array}{l}\mathrm{Nb} . \\
\text { foll. } \\
\text { totaux }\end{array}$ & $\begin{array}{c}\mathrm{Nb} . \\
\text { foll. } \\
\text { norm. }\end{array}$ & $\begin{array}{l}\text { m nb. } \\
\text { foll. } \\
\text { tot. }\end{array}$ & $\begin{array}{c}\text { ma nb. } \\
\text { foll. } \\
\text { norm. }\end{array}$ & $\begin{array}{l}\% \text { foll } \\
\text { atrét. }\end{array}$ & $\begin{array}{l}\text { Moy. } \\
\text { surf. } \\
\text { foll. } \\
\text { norm. }\end{array}$ & $\begin{array}{l}\text { Moy. } \\
\text { surf. } \\
\text { foll. } \\
\text { atrét. }\end{array}$ \\
\hline Total & 0,37 & 239 & 5,43 & 1642600 & 560 & 409 & 7,57 & 5,53 & 0,27 & 1447200 & 1270900 \\
\hline Test & & & $\leftarrow$ & $\longleftarrow$ & - NS - & - & $-*$ & $\rightarrow$ & & & \\
\hline Californienne & 0,37 & 127 & 6,68 & 1682300 & 279 & 211 & 8,45 & 6,39 & 0,24 & 1423000 & 1232800 \\
\hline Néo-Zélandaise & 0,38 & 112 & 4,48 & 1597700 & 281 & 198 & 6,85 & 4,83 & 0,30 & 1473000 & 1302200 \\
\hline Test & NS & & $* *$ & NS & & & $* *$ & ** & & NS & NS \\
\hline Nullipare & 0,33 & 71 & 4,73 & 1593100 & 209 & 153 & 6,97 & 5,10 & 0,27 & 1422400 & 1276600 \\
\hline Primipare & 0,40 & 89 & 5,56 & 1605000 & 188 & 136 & 7,83 & 5,67 & 0,28 & 1346300 & 1223500 \\
\hline Multipare & 0,39 & 79 & 6,08 & 1729500 & 163 & 120 & 8,15 & 6,00 & 0,26 & 1593200 & 1320900 \\
\hline Test & NS & & NS & NS & & & NS & NS & & $* *$ & NS \\
\hline Saison 1 & 0,37 & 62 & 6,20 & 1678200 & 129 & 91 & 7,59 & 5,35 & 0,29 & 1512300 & 1276900 \\
\hline Saison 2 & 0,29 & 50 & 5,00 & 1523100 & 183 & 137 & 7,62 & 5,71 & 0,25 & 1520300 & 1341300 \\
\hline Saison 3 & 0,36 & 61 & 5,55 & 1826800 & 146 & 106 & 7,68 & 5,58 & 0,27 & 1463900 & 1286700 \\
\hline Saison 4 & 0,48 & 66 & 5,08 & 1529500 & 102 & 75 & 7,29 & 5,36 & 0,26 & 1211200 & 1119500 \\
\hline Test & NS & & NS & ** & & & NS & NS & & $* *$ & NS \\
\hline
\end{tabular}

(1) p. $100=\frac{\text { Nambre de femelles à ovaires sains }}{\text { Nombre de femelles totales }}$

(2) Moyenne surface $=\mu m^{2}$.

Saison 1 : février ; Saison 2 : mai ; Saison 3 : août : Saison 4 : novembre.

[L'interaction saison-parité sur la surface des follicules normaux est hautement significative $\left({ }^{* *}\right)$; les autres interactions " diverses " sont non significatives (NS).]

maux. Ces proportions sont comparables pour les 2 génotypes quelles que soient la parité et la saison.

Le nombre moyen de follicules pré-ovulatoires chez les femelles ne montrant pas d'atrésie est identique $(5,43)$ à celui des follicules sains $(5,53)$ chez les femelles ayant des follicules sains et atrétiques, ces derniers représentant 25 à $30 \%$ des follicules.

Les follicules sains des femelles ne présentant pas d'atrésie sont significativement plus gros (1 $642600 \mu \mathrm{m}^{2}$; DE : 1,5 mm) que les follicules sains des femelles ayant aussi des follicules atrétiques (1 $447200 \mu \mathrm{m}^{2}$; DE : $1,3 \mathrm{~mm}$ ) quels que soient le génotype, la parité (bien que la taille moyerıne soit plus grande chez les 
multipares) et la saison (sauf en mai où les tailles sont comparables). La taille des follicules atrétiques est toujours inférieure à celle des follicules sains.

Les distributions sont significativement différentes (fig. 7). Les follicules de la population sans atrésie ont des tailles en moyenne plus homogènes et sont rassemblés essentiellement dans les classes 4 à 8 .

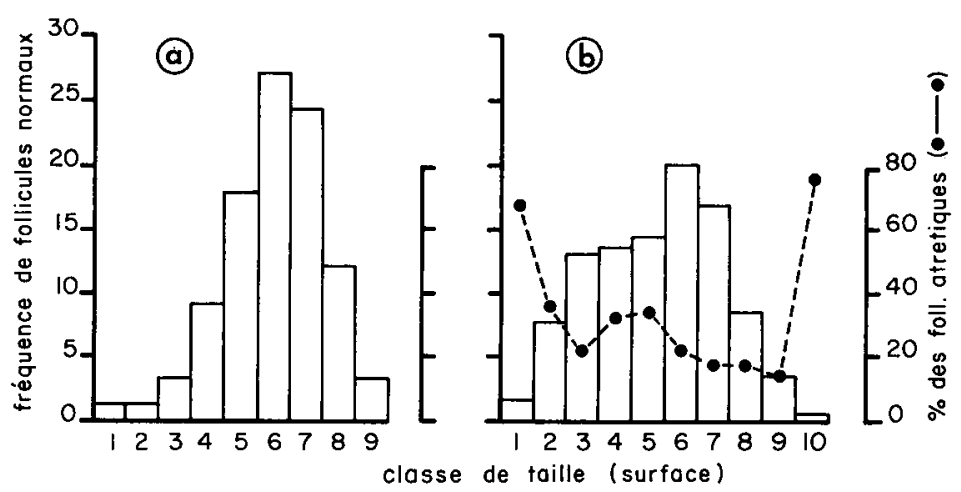

FIG. 7. - Distribution des follicules normaux dans les 2 catégories d'ovaires pour l'ensemble des femelles (Néo-Zélandaises + Californiennes). Evolution du pourcentage d'atrésie en fonction de la taille des follicules (a : ovaire avec follicules préovulatoires normaux; b : ovaire avec follicules préovulatoires normaux et atrétiques).

L'étude n'ayant porté que sur l'ovaire droit des femelles, nous avons étudié un échantillon d'ovaires gauches parmi les femelles n'ayant que des follicules sains sur l'ovaire droit. Sur 34 ovaires gauches étudiés, 21 présentent uniquement des follicules préovulatoires sains comme pour l'ovaire droit, 7 ovaires possèdent parmi tous les follicules sains 1 follicule atrétique et les 6 ovaires restants 2 à 3 follicules atrétiques, l'ensemble représentant un taux d'atrésie global de $9 \%$ (alors qu'il est de $27 \%$ dans le lot des femelles ayant des follicules sains et atrétiques). Nous en concluerons qu'il existe bien 2 groupes de femelles, l'un caractérisé par un taux d'atrésie nul ou faible, l'autre par un taux d'atrésie touchant un peu plus du quart des follicules préovulatoires.

\section{Discussion.}

1) Caractéristiques de la population des follicules préovulatoires chez la lapine. - Si l'étude chronologique de la croissance des follicules préovulatoires au cours du cycle cestrien ou après le coït a été suivie dans plusieurs espèces de rongeurs (rat, Boling et al., 1941 ; hamster, Norman et Greenwald, 1972 ; lapin, Thébault, 1977) une étude statistique des tailles des follicules manifestant une reprise de la méiose de leur ovocyte n'a jamais été réalisée. L'étude de la distribution des tailles des follicules, $8 \mathrm{~h}$ après le coït, montre que la décharge des gonadotropines touche une large gamme de follicules à différents stades de croissance et synchronise la reprise de la méiose, conduisant au stade préovulatoire des follicules dont la surface peut varier dans des proportions de 1 à 6 . 
Mariana (1984) a montré chez la rate que les follicules reprenant la méiose pouvaient avoir des diamètres allant de $263 \mu \mathrm{m}$ à $800 \mu \mathrm{m}$ environ, ce qui confirme la grande variabilité de taille caractérisant les follicules préovulatoires des espèces polytoques.

On observe également une certaine plasticité de la reprise de la méiose en fonction de la pycnose cellulaire. En effet, des follicules qui viennent d'ovuler $10 \mathrm{~h}$ après le coït, peuvent contenir jusqu'à 20 cellules pycnotiques. Ceci pose le problème de la qualité des ovocytes issus de tels follicules et de leur viabilité ultérieure (lapine, Thébault, 1977 ; rate, Peluso et Downey, 1982).

Le taux d'atrésie est plus important dans les classes de follicules préovulatoires de petites tailles, ce qui peut signifier qu'ils sont plus sensibles à l'atrésie ou que leur croissance est freinée. Hirshfield et Midgley (1978) note chez la rate un stade critique dans la croissance où l'atrésie joue un rôle important : les follicules ayant un diamètre compris entre 200 et $400 \mu \mathrm{m}$ (petits préovulatoires) ont un taux d'atrésie plus marqué qu'à d'autres stades de développement. Quant à un éventuel ralentissement de la croissance, il n'est pas prouvé. L'index mitotique des cellules de la granulosa n'est pas ralenti au début de l'atrésie (Byskov, 1974). La perte des récepteurs (Richards, 1980) pourraient entraîner toutefois un ralentissement des sécrétions stéroïdiennes et un développement moindre de l'antrum.

2) Effet des facteurs d'environnement : parité-saison. L'absence d'interaction statistique entre le génotype et les facteurs d'environnement permet de conclure que ces derniers modifient les paramètres de la croissance folliculaire de la même manière dans les deux souches. Ces souches sont donc en toutes circonstances, identifiables à partir de leurs caractéristiques ovariennes (nombre d'ovulations et de follicules préovulatoires).

II est bien montré que dans les différentes espèces animales, et particulièrement la lapine, le nombre de petits nés augmente au cours des premières portées (Larson et al., 1973), et varie au cours des saisons (Sittman et al., 1964). Nous pensons, au vu de notre étude, que cet accroissement est la conséquence d'une augmentation du nombre et de la taille des follicules préovulatoires sains (environ +2 , pour les deux ovaires entre nullipares et multipares), l'atrésie étant un phénomène secondaire d'ampleur relativement constante en toute circonstance. Ceci entraîne un accroissement du nombre d'ovulations $(+2,72$ entre les mêmes stades : Hulot et Matheron, 1981) responsable d'une meilleure prolificité. Les travaux sur la souris de Pedersen (1972), Faddy et al. (1976, 1983) permettent d'expliquer ces résultats. Ils ont montré, en effet, que la vitesse de croissance des follicules variait avec le stade de leur développement mais aussi durant la vie de l'animal. L'augmentation du nombre de follicules avec l'âge correspond sans doute à une accélération de la vitesse de croissance et à une accumulation des follicules vers les tailles supérieures à $500000 \mu \mathrm{m}^{2}$ (DE : $0,8 \mathrm{~mm}$ ). Les stades nullipares (4,5 mois) et primipares (6 à 6,5 mois) correspondraient à une certaine « jeunesse 》 de l'animal, l'ovaire atteignant sa complète maturité chez la femelle multipare âgée de 9-10 mois. Il existe également un effet saisonnier caractérisé par une variation de la taille des follicules des lapines adultes. Pendant les mois de plus faible activité sexuelle chez la lapine (octobre-novembre, Thibault et al., 1966), la taille des follicules préovulatoires des femelles multipares dans les deux souches 
est plus petite. Chez les jeunes lapines (nullipares et primipares) des deux génotypes, on est en toute saison à la taille critique moyenne minimale.

3) Caractéristiques des deux types génétiques. - La lapine de race Californienne présente un plus grand nombre de follicules préovulatoires que la lapine de race Néo-Zélandaise ; le même type d'observations a été fait sur les brebis de prolificités différentes (Turnbull et al., 1978 ; Cahill et al., 1981; Lahlou-Kassi et Mariana, 1984).

Hormis ce fait, les taux d'atrésie sont peu différents entre ces deux souches et la répartition des follicules en classes de taille est semblable (mêmes classes extrêmes, même taille moyenne). On peut en conclure que pour les femelles des deux souches, les mécanismes assurant la croissance terminale des follicules précédant l'ovulation, sont comparables. II n'a pas été mis non plus en évidence de différence significative dans les taux circulant de LH et FSH pendant la période des pics pré et post ovulatoires chez ces deux souches (bien que les pics préovulatoires paraissent légèrement plus élevés chez la femelle Néo-Zélandaise) (Meunier et al., 1983). On doit donc rechercher l'origine de la différence du nombre des follicules préovulatoires à un stade plus précoce de la croissance folliculaire, par exemple, au moment de la formation de la population des follicules primordiaux et du démarrage de leur croissance au cours du premier mois de la vie de la femelle.

La comparaison des nombres et des tailles des follicules préovulatoires sains chez les deux souches d'une part et entre les femelles nullipares et multipares d'autre part, montre que des mécanismes différents en apparence peuvent entraîner un accroissement du nombre des follicules. Dans les deux souches, bien que les distributions des tailles des follicules soient identiques, leur nombre est différent. II semble cependant que l'augmentation du nombre des follicules chez les multipares résulte d'un changement de la vitesse de croissance lié sans doute à des niveaux circulants de FSH plus importants (rate, Peluso et Downey, 1982) conduisant à des follicules plus gros.

4) Femelles ne reprenant pas la méiose. - $23 \%$ des femelles n'ovuleront pas ; ces femelles présentent le même nombre de follicules totaux (sains et atrétiques) et que les femelles qui ovuleront, mais leurs follicules ont une taille moitié moindre environ, pour les deux souches (environ $800000 \mu \mathrm{m}^{2}$; DE : $1 \mathrm{~mm}$ ). La différence peut représenter l'accroissement de taille obtenu, $8 \mathrm{~h}$ après le coït sous l'action des décharges d'hormones gonadotropes. Cependant, les travaux de Thébault (1977) indiquent que la taille moyenne des follicules au moment du coït est de l'ordre de 1,2 à 1,3 mm. Par ailleurs, des mesures de taille de follicules sains réalisées sur des ovaires de femelles mises au mâle 1 h 30 plus tôt, ont révélé l'existence de 2 types de femelles : des femelles ayant des follicules de diamètre supérieur à $1,20 \mathrm{~mm}$ et des femelles ayant des petits follicules d'un diamètre voisin de $1 \mathrm{~mm}$, les coefficients de variation des tailles étant les mêmes dans les 2 cas, ce qui suggère que les follicules de grande taille sont renouvelées de façon synchrone. Il est classique d'admettre que le coït par la voie d'un réflexe neurohypothalamique provoque la décharge de $\mathrm{LH}$ et l'ovulation. Des femelles n'ovuleraient pas à la suite du coït, faute de décharge de LH (la cause de la non- 
décharge de cette hormone est peut être associée à un niveau insuffisant des sécrétions œstrogéniques chez les femelles à petits follicules).

Le taux d'atrésie plus élevé des femelles n'ovulant pas est certainement lié à la non-décharge du 1er pic de FSH (Braw et Tsafriri, 1980).

5) L'atrésie. - Nous avons mis en évidence deux types de femelles; celles dont les ovaires ont une population de follicules de grande taille ne présentant, pour la plupart, aucun signe d'atrésie, et les femelles présentant un taux d'atrésie global voisin de $27 \%$.

Dans les deux cas, le nombre des follicules sains est comparable. On peut faire I'hypothèse que l'atrésie serait un élément de régulation tardif du nombre de follicules préovulatoires et participerait ainsi indirectement à la régulation du taux d'ovulation.

En outre, les follicules préovulatoires des femelles sans atrésie sont de plus grande taille. Il semble exister, là encore, un système biologique de régulation au niveau de la folliculogenèse, indépendant du génotype et des facteurs environnants. L'absence d'atrésie et la plus grande taille des follicules résultent peut-être d'un niveau circulant de FSH plus important, protégeant les follicules et accélérant simultanément leur croissance.

Un rapprochement intéressant peut être établi entre le pourcentage de femelles ne présentant pas d'atrésie des gros follicules $(37 \%)$ et le pourcentage des femelles ovulant ne présentant pas de mortalité préimplantatoire $(29 \%)$ (Hulot et Matheron, 1981). Ces deux pourcentages ne diffèrent pas significativement. II reste à démontrer que les femelles possédant presque exclusivement des follicules préovulatoires sains sont aussi celles qui ont une très bonne viabilité des blastocystes.

Reçu en février 1983. Accepté en août 1984.

\section{Références}

BOLING J. L., BLANDAU R. J., SODERWALL A. L., YOUNG W. C., 1941 . Growth of the Graafian follicle and the time of ovulation in the albino rat. Anat. Rec., 79, 313-331.

BRAW R., TSAFRIRI A., 1980. Follicles explanted from pentobarbitone treated rats provide a model for atresia. J. Reprod. Fert., 59, 259-265.

BYSKOV A. G., 1974. Cell. Kinetic studies of follicular atresia in the mouse ovary. J. Reprod. Fert., 37, 277-285.

CAHILL L. P., SAUMANDE J., RAVAULT J. P., BLANC M., THIMONIER J., MARIANA J. C., MAULÉN P., 1981. Hormonal and follicular relationships in ewes of high and low ovulation rates. J. Reprod. Fert, 62, 141-150.

CHERNEY D. D., DIDIO L. J. A., MOTTA P., 1975. The development of rabbit ovarian follicles following copulation. Fert. Ster., 26, 257-270.

FADDY M. J., JONES E., EDWARDS R. G., 1976. An analytical model for ovarian follicle dynamics. J. exper. Zool., 197, 173-185.

FADDY M. J., GOSDEN R. G., EDWARDS R. G., 1983. Ovarian follicle dynamics in mice : a comparative study of three inbred strains and F1 hybrid. J. Endocr., 96, 23-33.

HARVEY W. R., 1975. Least square analysis of data with unequal subclas numbers. Agr. Res. Ser. US Dept. Agr., 157 pp. 
HIRSHFIELD A. N., MIDGLEY A. R., 1978. Morphometric analysis of follicular development in the rat. Biol. Reprod., 19, 597-605.

HULOT F., MATHERON G., 1981. Effet du génotype de l'âge et de la saison sur les composantes de la reproduction chez la lapine. Ann. Génét. Sél. anim., 13, 131-150.

LAHLOU-KASSI A., MARIANA J. C., 1984. Ovarian follicular growth during the oestrous cycle in two breeds of ewes of different ovulation rate. The D'Man and the Timahdite. J. Reprod. Fert. (à paraître).

LARSON L. L., SPILMAN C. H., DUNN H. A., FOOTE R. H., 1973. Reproductive efficiency in aged female rabbits given supplemental progesterone and oestradiol. J. Reprod. Fert., 33, $31-38$.

MARIANA J. C., 1984. Croissance folliculaire normale et provoquée chez la rate 33-45. In SALATBAROUX J., THIBAULT Ch., Période péri-ovulatoire, Coll. Soc. franç. pour l'étude de la fertilité. Masson, Paris.

MAULÉON P., MARIANA J. C., 1977. Oogenesis and folliculogenesis, 175-202. In COLE H. H., CUPPS P. T., Reproduction in domestic animals (3rd edition) Acad. Press, New York, San Francisco, London.

MAULÉON P., RAO K. H., 1963. Variations génétiques des populations folliculaires dans les ovaires de rates impubères. Ann. Biol. anim. Bioch. Biophys., 3, 21-31.

MEUNIER M., HULOT F., POIRIER J. C., TORRĖS S., 1983. A comparison of ovulatory gonadotrophic surge in two rabbit strains: no évidence for a relationship between LH or FSH surge and factors of prolificacy Reprod. Nutr. Dévelop., 23, 709-715.

NORMAN R. L., GREENWALD G., 1972. Follicular histology and physiological correlates in the preovulatory hamster. Anat. Rec., 173, 95-108.

PEDERSEN T., 1972. Follicle growth in the mouse ovary, 261-276. In BIGGERS J. D., SCHUETZ A. W., Oogenis, Univ. Park Press, Baltimore. Butterworth, London.

PELUSO J. J., DOWNEY C., 1982. Pattern of follicular development during the estrous cycle of aged rats. Cell Tissue Res., 225, 229-234.

PETERS H., McNATTY K. P. 1980. The ovary. Reproduct. Biol. Handbooks, Univ. Liverpool. C. A. FINN Ed. Granada Publ. London. 175 pp.

RICHARDS J., 1980. Maturation of ovarian follicles : actions and interactions of pituitary and ovarian hormones on follicular cell differenciation Physiol. Rev., 60, 51-89.

SITTMANN D. B., ROLLINS W. C., SITTMANN K., CASADY R. R. 1964. Seasonal variation on reproductive traits of the New-Zealand white rabbit $J$. Reprod. Fert., 8, 29-37.

SOKAL R., ROHLF F. J., 1969. Biometry. The principles and practice of statistics in biological research. W. H. Freeman and Co. San Francisco.

THÉBAULT (A.), 1977. Maturation ovocytaire et activité de la granulosa dans le follicule préovulatoire chez la lapine. Diplôme d'études approfondies de Physiologie de la Reproduction. Université Pierre et Marie Curie.

THIBAULT C., COUROT M., MARTINET L., MAULÉON P., du MESNIL du BUISSON F., ORTAVANT R., PELLETIER J., SIGNORET J. P., 1966. Regulation of breeding season and oestrus cycles by light and external stimuli in some mammals. J. anim. Sci., 25 (suppl. 1), 119-142.

THIBAULT C., GÉRARD M., MÉNÉZO Y., 1976. Nuclear and cytoplasmic aspects of mammalian oocyte maturation in vitro in relation to follicle size and fertilization. Sperm Action. Prog. Reprod. Biol., 1, 233-240 (Karger, Basel).

TURNBULL K. E., MATTNER P. E., GEORGE J. M., SCARAMUZZ| R. J., 1978. The relation between patterns of ovarian follicle growth and ovulation rate in sheep. Aust. J. biol. Sci., 31, 649-655. 